This is the accepted manuscript made available via CHORUS. The article has been published as:

Unusual phonon density of states and response to the superconducting transition in the In-doped topological crystalline insulator Pb_\{0.5\}Sn_\{0.5\}Te

Kejing Ran, Ruidan Zhong, Tong Chen, Yuan Gan, Jinghui Wang, B. L. Winn, A. D. Christianson, Shichao Li, Zhen Ma, Song Bao, Zhengwei Cai, Guangyong Xu, J. M. Tranquada, Genda Gu, Jian Sun, and Jinsheng Wen

Phys. Rev. B 97, 220502 - Published 15 June 2018 DOI: 10.1103/PhysRevB.97.220502 


\title{
Unusual phonon density of states and response to superconducting transition in In-doped topological crystalline insulator $\mathrm{Pb}_{0.5} \mathrm{Sn}_{0.5} \mathrm{Te}$
}

\author{
Kejing Ran, ${ }^{1}$ Ruidan Zhong, ${ }^{2,3}$, * Tong Chen, ${ }^{1}$ Yuan Gan, ${ }^{1}$ Jinghui Wang, ${ }^{1}$ \\ B. L. Winn, ${ }^{4}$ A. D. Christianson, ${ }^{4}$ Shichao Li,${ }^{1}$ Zhen Ma, ${ }^{1}$ Song Bao, ${ }^{1}$ Zhengwei Cai, ${ }^{1}$ \\ Guangyong Xu, ${ }^{5}$ †. M. Tranquada, ${ }^{5}$ Genda Gu, ${ }^{5}$ Jian Sun,,${ }^{1,6}$, 声 and Jinsheng Wen ${ }^{1,6}$, 8 \\ ${ }^{1}$ National Laboratory of Solid State Microstructures and Department of Physics, Nanjing University, Nanjing 210093, China \\ ${ }^{2}$ Condensed Matter Physics and Materials Science Department, \\ Brookhaven National Laboratory (BNL), Upton, New York 11973, USA \\ ${ }^{3}$ Materials Science and Engineering Department, \\ Stony Brook University, Stony Brook, New York 11794, USA \\ ${ }^{4}$ Neutron Scattering Division, Oak Ridge National Laboratory (ORNL), Oak Ridge, Tennessee 37831, USA. \\ ${ }^{5}$ Condensed Matter Physics and Materials Science Department, \\ Brookhaven National Laboratory, Upton, New York 11973, USA \\ ${ }^{6}$ Collaborative Innovation Center of Advanced Microstructures, Nanjing University, Nanjing 210093, China
}

We present inelastic neutron scattering results of phonons in $\left(\mathrm{Pb}_{0.5} \mathrm{Sn}_{0.5}\right)_{1-x} \mathrm{In}_{x}$ Te powders, with $x=0$, and 0.3 . The $x=0$ sample is a topological crystalline insulator, and the $x=0.3$ sample is a superconductor with a bulk superconducting transition temperature $T_{c}$ of $4.7 \mathrm{~K}$. In both samples, we observe unexpected van Hove singularities in the phonon density of states at energies of 1$2.5 \mathrm{meV}$, suggestive of local modes. On cooling the superconducting sample through $T_{c}$, there is an enhancement of these features for energies below twice the superconducting-gap energy. We further note that the superconductivity in $\left(\mathrm{Pb}_{0.5} \mathrm{Sn}_{0.5}\right)_{1-x} \operatorname{In}_{x}$ Te occurs in samples with normal-state resistivities of order $10 \mathrm{~m} \Omega \mathrm{cm}$, indicative of bad-metal behavior. Calculations based on density functional theory suggest that the superconductivity is easily explainable in terms of electron-phonon coupling; however, they completely miss the low-frequency modes and do not explain the large resistivity. While the bulk superconducting state of $\left(\mathrm{Pb}_{0.5} \mathrm{Sn}_{0.5}\right)_{0.7} \mathrm{In}_{0.3} \mathrm{Te}$ appears to be driven by phonons, a proper understanding will require ideas beyond simple BCS theory.

Topological insulators (TIs) represent an exotic state of matter in which the bulk is insulating but the surface is metallic 12 . The topological state is protected by the time-reversal symmetry ${ }^{1}$. By including certain crystal point group symmetry instead of time reversal, topological crystalline insulators (TCIs), a state analogous to TIs, was also proposed ${ }^{3 / 4}$. In particular, it was predicted that compounds such as SnTe might be TCIs ${ }^{5}$, and the key features, including the inverted character of bands near the chemical potential ${ }^{6 / 7}$ and surface states within the band gap that are protected from back-scattering 8 , were soon verified.

These developments have also spurred renewed interest in topological superconductors, as such materials may exhibit gapless surface states that could be beneficial for quantum computing 1.3. A common aspect of TCIs is strong spin-orbit coupling in the atomic states contributing to the valence and conduction bands, and this is a useful ingredient for obtaining the unusual pairing symmetry expected for a topological superconductor. Hence, there is interest in seeing whether doping TCIs can induce superconductivity with a topological nature. Indeed, one can make SnTe superconducting by introducing In, and a point-contact study found evidence of surface Andreev bound states 10 , though thermodynamic studies suggest a fully gapped superconducting state 11 .

$\mathrm{SnTe}$ is a compound that has the rocksalt structure despite the fact that the component elements do not all form closed-shell ions. A consequence is a strong intrinsic electron-phonon coupling ${ }^{14115}$, which leads to a ferro- electric phase transition in $\mathrm{SnTe}^{16117}$. One way to suppress the ferroelectric transition is to substitute $\mathrm{Pb}$ for Sn, and it has been estimated that the transition should hit $0 \mathrm{~K}$ for $\mathrm{Pb}_{y} \mathrm{Sn}_{1-y} \mathrm{Te}$ with $y \approx 0.5 \frac{18}{10}$. At this $\mathrm{Pb}$ concentration the system becomes a TCl19. Also, substitution of a small amount of In turns the system into a true bulk insulator ${ }^{20}$, while adding more In makes it superconducting 21222. For reference, we note that the proximity of the superconductivity and the suppressed ferroelectric order suggests a possible connection with the enhanced superconductivity at the ferroelectric quantum critical point in $\mathrm{SrTiO}_{3}{ }^{23 / 24}$.

In this Rapid Communications, we use inelastic neutron scattering (INS) to measure the phonon density of states (PDOS) in polycrystalline samples of $\left(\mathrm{Pb}_{0.5} \mathrm{Sn}_{0.5}\right)_{1-x} \mathrm{In}_{x} \mathrm{Te}$ (PSIT) with $x=0$ (a nonsuperconducting TCI), and $x=0.3$ (a superconductor with a critical temperature $T_{c}$ of $4.7 \mathrm{~K}$ ). In both samples, we detect unexpected peaks, in the range of 1 to 2.5 $\mathrm{meV}$, that are not reproduced by a calculation using the virtual crystal approximation. While calculations of the electron-phonon coupling indicate contributions from phonon features from 3 to $12 \mathrm{meV}$ that should easily be sufficient to explain the $T_{c}$, we observe a surprising enhancement below $T_{c}$ of phonon intensities with energies less than twice that of the $T=0$ superconducting gap. We also note that the normal-state resistivity is unusually large and nonmetallic, possibly associated with the electron-phonon coupling; in any case, it raises questions regarding the existence of well-defined quasiparticles and 

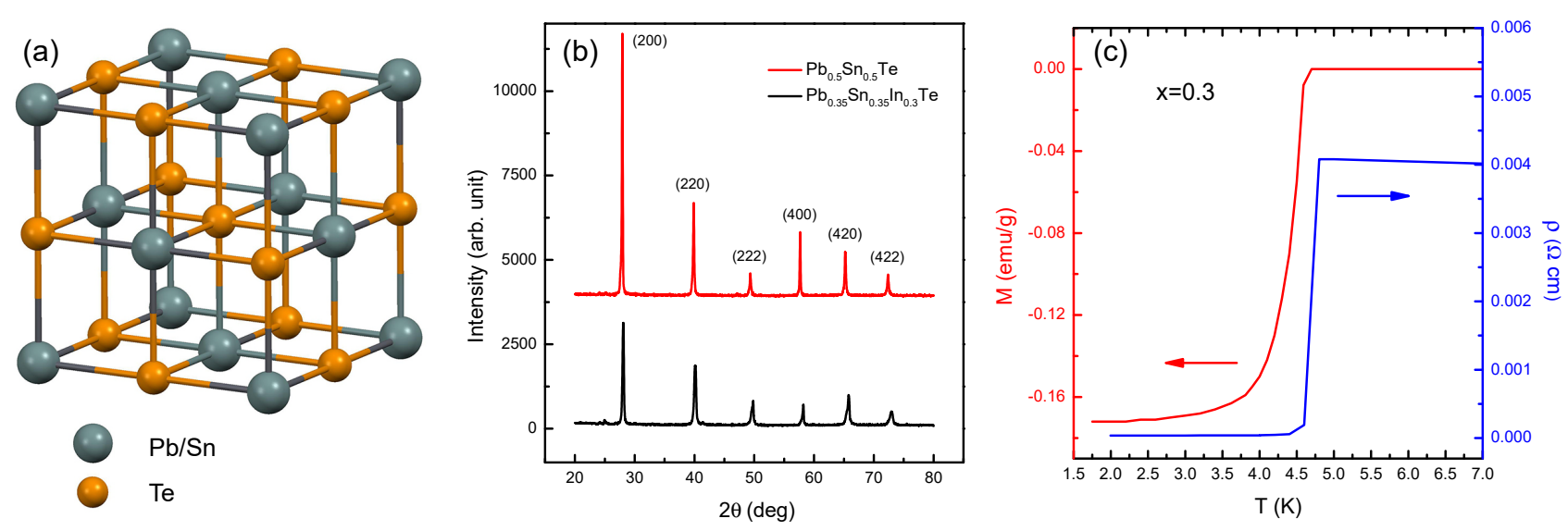

FIG. 1. (a) Schematic for the crystal structure of $\mathrm{Pb}_{0.5} \mathrm{Sn}_{0.5}$ Te. (b) X-ray powder diffraction results for $\left(\mathrm{Pb}_{0.5} \mathrm{Sn}_{0.5}\right)_{1-x} \mathrm{In}_{x} \mathrm{Te}$ with $x=0$ and 0.3 measured at room temperature. (c) Temperature dependence of the magnetization (left axis) and resistivity (right axis) for $\left(\mathrm{Pb}_{0.5} \mathrm{Sn}_{0.5}\right)_{0.7} \mathrm{In}_{0.3}$ Te single crystals. The magnetization were measured in zero-field-cooling conditions with a magnetic field of 10 Oe.

the applicability of conventional BCS theory 25 .

Single crystals of PSIT with $x=0$ and 0.3 were grown by the modified floating-zone technique as described in refs $22|26| 27$. The sample compositions were measured with a scanning electron microscope (SEM) equipped with an analyzer for energy dispersive X-ray spectroscopy. About 10 positions were measured for each crystal piece, and the variation in $x$ was generally found to be $<2 \%$ of the mean value. Moreover, the SEM images showed that there is no secondary phase for the samples studied here ${ }^{22}$. Fine powders were obtained by grinding these crystals inside a glovebox filled with Ar. Powder x-ray diffraction (XRD) measurements were performed at room temperature on a Rigaku Miniflex II located at Center for Functional Nanomaterials at BNL, using $\mathrm{Cu} K \alpha$ radiation. The diffraction patterns, plotted in Fig. 1(b), can be nicely indexed with the rocksalt structure (space group $F m \overline{3} m$ ) shown in Fig. 1(a). These data indicate that impurity level is below 1\%. Roomtemperature lattice constants are determined to be 6.392 and $6.359 \AA$ for the $x=0$, and 0.3 samples, respectively.

Magnetization and resistivity were measured on singlecrystal pieces extracted from the same batches as those used to make the powders for XRD and INS measurements. Magnetization and resistivity were measured using a Quantum Design Magnetic Properties Measurement System and Physical Properties Measurement System, respectively. The sample with no $\mathrm{In}$ is a $\mathrm{TCl}^{22 \mid 27 / 28}$. It shows a weakly metallic behavior and is not superconducting down to $1.8 \mathrm{~K}$. The $x=0.3$ sample is superconducting with a $T_{c}$ of $4.7 \mathrm{~K}$, as determined from the onset of diamagnetism and zero resistivity, as shown in Fig. 1(c). This is the optimal $T_{c}$ of this system, before the solubility of $x=0.35$ is reached 2227 . The $x=0.3$ sample shows sharp superconducting transition in both the magnetization and resistivity measurements, indicating that the sample is homogenous, consistent with the
$\mathrm{XRD}$ results and composition analysis.

We used 20-g powders well characterized as discussed above for each compound in the INS experiments, carried out on two time-of-flight (TOF) spectrometers HYSPEC 29 and ARCS, both located at the Spallation Neutron Source (SNS) at ORNL. On HYSPEC and ARCS, we chose an incident energy $E_{i}$ of 15 and $35 \mathrm{meV}$, respectively. On HYSPEC, we used a Fermi frequency of $360 \mathrm{~Hz}$, which gave an energy resolution of $\sim 0.4 \mathrm{meV}$ (full
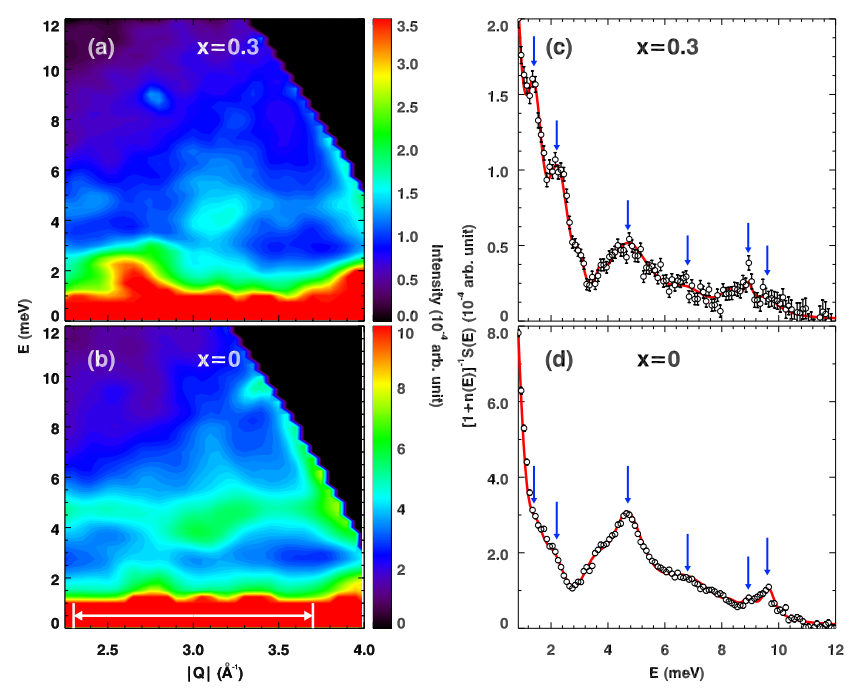

FIG. 2. (a) and (b) Inelastic neutron scattering results for $\left(\mathrm{Pb}_{0.5} \mathrm{Sn}_{0.5}\right)_{1-x} \operatorname{In}_{x}$ Te with $x=0.3$ and 0 , respectively, measured at $7 \mathrm{~K}$; (c) Scattering intensities (corrected by the Bose factor) as a function of energy obtained by integrating the intensities in (a) with $|\boldsymbol{Q}|$ ranging from 2.2 to $3.7 \AA^{-1}$ [indicated in (b)] for $x=0.3$ sample; (d) Same as (c), but for the $x=0$ sample. Lines through data are guides to the eye. Vertical arrows represent major phonon modes. Error bars represent one standard deviation throughout the paper. 
width at half maximum) at the elastic position. Only the low-energy data collected on HYSPEC are presented in this work. The results at $7 \mathrm{~K}$ (above the $T_{c}$ of the superconducting sample) are shown in Fig. 2(a) and (b). Since In absorbs neutrons strongly $\frac{30}{3}$, the scattering intensities for the In-doped sample are much weaker than those of the In-free sample. The scattering around $5 \mathrm{meV}$ is prominent, with the strength increasing with increasing $|\boldsymbol{Q}|$, where $\boldsymbol{Q}$ is the wave vector. Such a $\boldsymbol{Q}$ dependence of the scatteried intensity is consistent with those resulting from phonons. We integrate the intensities $S(\boldsymbol{Q}, E)$ over $\boldsymbol{Q}$ ranging from 2.2 to $3.7 \AA$, as indicated in Fig. 2 (b), divide them by the detailed-balance factor $[1+n(E)]$, where $n(E)$ is the Bose distribution function, and plot them as a function of energy in Fig. 2(c) and (d). Prominent features are indicated by the arrows in Fig. 2(c) and (d). Although the positions for both samples appear to be the same, the relative intensities among different features show some differences. These indicate that although the In doping does not alter the crystal structure significantly, it does affect the lattice dynamics.

The measurements on the $x=0.3$ sample were repeated at $1.5 \mathrm{~K}$, well below $T_{c}$. The only significant changes across $T_{c}$ occur at low energies, as illustrated in Fig. 3. At $1.5 \mathrm{~K}$, the intensities are enhanced compared to those at $7 \mathrm{~K}$ for energies below $\sim 1.4 \mathrm{meV}$. Interestingly, this energy is almost exactly twice of the zerotemperature superconducting gap, $2 \Delta(0)=1.44 \mathrm{meV}$ obtained in the same material from scanning tunneling

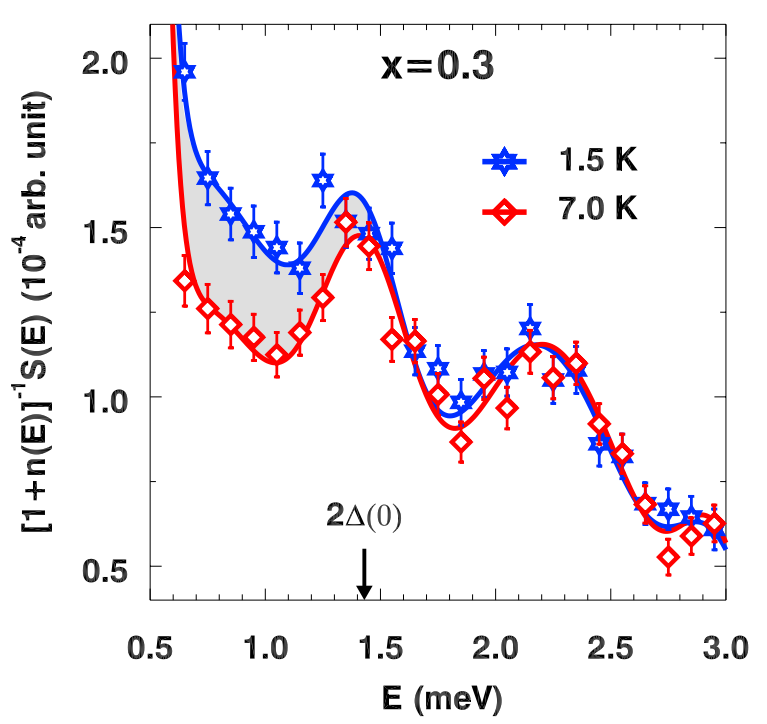

FIG. 3. Low-energy phonon spectra for $\left(\mathrm{Pb}_{0.5} \mathrm{Sn}_{0.5}\right)_{0.7} \mathrm{In}_{0.3} \mathrm{Te}$ at 1.5 and $7 \mathrm{~K}$. Intensities $S(E)$ obtained by integrating the intensities in Fig. 2 (a) with $|\boldsymbol{Q}|$ ranging from 2.5 to $3.0 \AA^{-1}$ have been corrected by the Bose factor. The arrow indicates twice of the estimated zero-temperature superconducting gap, $2 \Delta(0)$. Lines through data are guides to the eye. The shadow illustrates the difference between the low- and high-temperature data. spectroscopy measurements 31 . The ratio of $2 \Delta(0)$ over $k_{\mathrm{B}} T_{c}$ is about 3.5 , consistent with the BCS prediction, corresponding to the weak-coupling regime ${ }^{25}$. This ratio is also consistent with that obtained in a similar system, $\mathrm{Sn}_{1-x} \mathrm{In}_{x} \mathrm{Te}^{32}$. For reference, the non-superconducting $\mathrm{Pb}_{0.5} \mathrm{Sn}_{0.5} \mathrm{Te}$ sample has also been measured at $1.5 \mathrm{~K}$, but there was no significant change relative to $7 \mathrm{~K}$. The enhancement of the phonon scattering only in the superconducting sample below $T_{c}$, with a characteristic energy equal to $2 \Delta(0)$, indicates an intriguing and highly unusual correlation between the low-energy phonons and the superconducting order.

To gain further insight, we have converted the $|\boldsymbol{Q}|$ integrated scattered intensities $S(E)$ into the PDOS $G(E)=\sum_{i} \frac{\sigma_{i}}{m_{i}} \mathrm{e}^{-2 W_{i}} G_{i}(E)$, where $\sigma_{i}, m_{i}, W_{i}$, and $G_{i}$ are the neutron scattering cross section, atomic mass, Debye-Waller factor, and partial PDOS of the $i$ th atom 33 . $S(\boldsymbol{Q}, E)$ is related to $G_{i}(E)$ by

$$
S(\boldsymbol{Q}, E)=\sum_{i} \sigma_{i} \frac{\hbar|\boldsymbol{Q}|^{2}}{2 m_{i}} \mathrm{e}^{-2 W_{i}} \frac{G_{i}(E)}{E}[1+n(E)] .
$$

The resulting weighted PDOS for both samples at $T=$ $7 \mathrm{~K}$ are shown in Fig. 4(a). It is clear that there are some differences for the two samples, especially at energies below $3 \mathrm{meV}$. For the superconducting sample, $G(E)$ appears to be significantly higher than that of the nonsuperconducting sample at low energies.

To provide context for these experimental observations, we have performed phonon and electron-phonon coupling calculations in the framework of the density functional perturbation theory 34 . First-principles calculations were performed using the Quantum-Espresso $\operatorname{code} e^{35}$. Ultrasoft pseudopotentials with Perdew-Zunger type ${ }^{36}$ local density approximation exchange correlations were generated by virtual crystal approximation method 37 . Energy cutoffs of $100 \mathrm{Ry}$ for wave functions and 600 Ry for the charge density were employed. The self-consistent calculations were performed over a $12 \times 12 \times 12 k$-point grid. A denser $28 \times 28 \times 28 k$-grid was used for evaluating an accurate electron-phonon interaction matrix. Dynamical matrices and the electronphonon coupling were calculated on a $4 \times 4 \times 4 \boldsymbol{Q}$-point mesh.

The calculated PDOS for both the parent and doped samples are shown in Fig. 4(b). Overall, the peaks found in the calculations give a good description (after a slight energy scaling) of many features of the experimental results. The calculated isotropic electron-phonon coupling constant $\lambda$ for $\left(\mathrm{Pb}_{0.5} \mathrm{Sn}_{0.5}\right)_{0.7} \mathrm{In}_{0.3} \mathrm{Te}$ is about 1.4. We use the Allen-Dynes modification of the McMillan equa$\operatorname{tion}{ }^{38}, T_{c}=\omega_{\ln } / 1.2 \exp \left[-1.04(1+\lambda) / \lambda-\mu^{*}(1+0.62 \lambda)\right]$, where $\omega_{\ln }$ is the prefactor, and $\mu^{*}$ is the Coulomb pseudopotential, with a typical value of 0.13 . The $T_{c}$ is estimated to be around $7.6 \mathrm{~K}$, indicating that electronphonon coupling is, in principle, compatible with the actual $T_{c}$.

But is there anything wrong with this story? To begin with, the calculated PDOS's completely miss the exper- 

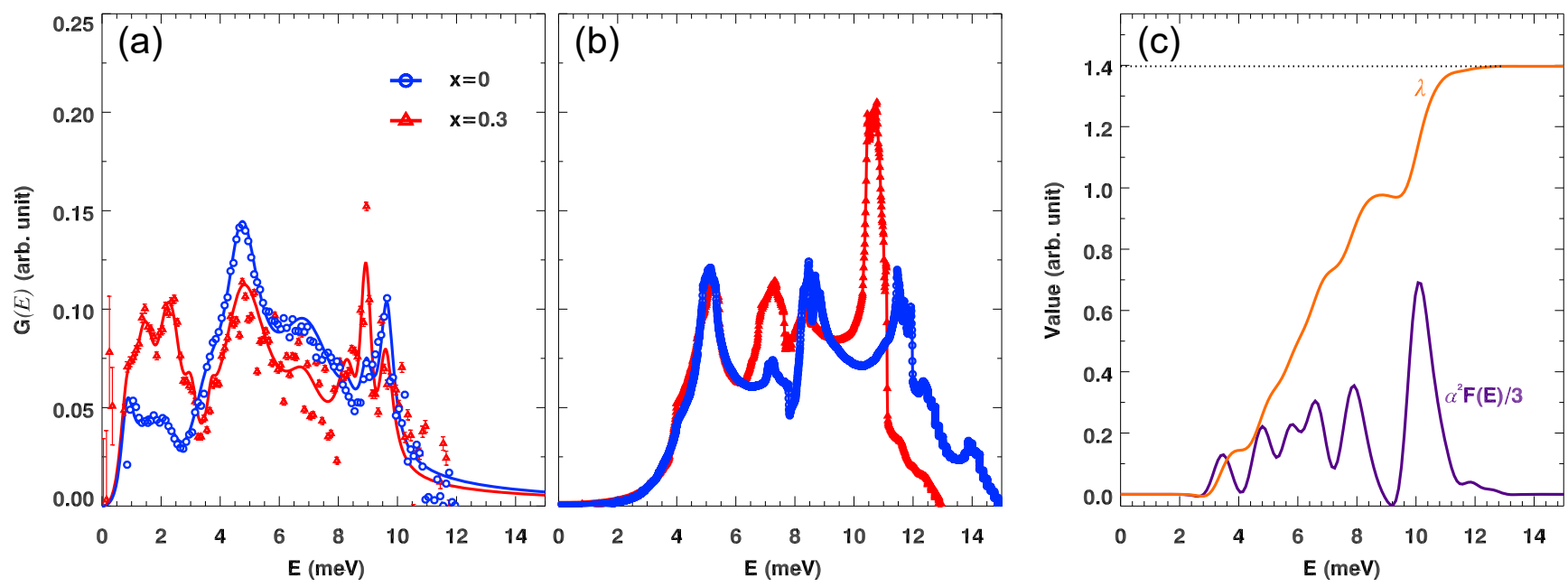

FIG. 4. (a) Experimental results of the weighted PDOS for $\left(\mathrm{Pb}_{0.5} \mathrm{Sn}_{0.5}\right)_{1-x} \operatorname{In}_{x} \mathrm{Te}$ with $x=0$ and 0.3 , measured at $7 \mathrm{~K}$. Lines through data are guides to the eye. (b) Calculated results of the PDOS for the two samples. (c) The calculated Eliashberg spectral function $\alpha^{2} F(E)$ for $\left(\mathrm{Pb}_{0.5} \mathrm{Sn}_{0.5}\right)_{0.7} \operatorname{In}_{0.3} \mathrm{Te}$, scaled by $1 / 3$, and the total electron-phonon coupling constant $\lambda$. The dashed line indicates the $\lambda$ value of 1.4 .

imental peaks below $3 \mathrm{meV}$. To appreciate this discrepancy, we first need to acknowledge that the peaks in the PDOS are van Hove singularities that occur when the derivative of a phonon frequency with respect to wave vector goes to zero. This typically occurs at a zone boundary, which then involves a substantial volume fraction of the Brillouin zone. The lowest-energy van Hove singularity tends to correspond to the zone-boundary energies of transverse acoustic (TA) phonons. In $\mathrm{PbTe}$ ( $\mathrm{SnTe})$, the TA mode at the $(1,1,0)$ zone-boundary point has an energy of about $4 \mathrm{meV}(6 \mathrm{meV})$ at $T=50 \mathrm{~K}^{39140}$. The average of these values, $5 \mathrm{meV}$, corresponds to the first big peak in Fig. 4(b) and a large peak in Fig. 4(a). Our mystery peaks are far below this.

The ferroelectric instabilities in both SnTe and $\mathrm{SrTiO}_{3}$ involve a soft, zone-center, transverse optical phonon 174142 . Such a mode would be unlikely to yield a significant peak in the PDOS, because the zone-center involves a small fraction of the Brillouin-zone volume. In our case, however, the mixture of $\mathrm{Pb}$ and $\mathrm{Sn}$ (and In) on the same face-centered-cubic sublattice breaks the symmetry of the rocksalt crystal structure, and may allow a mixing of zone-center and zone-boundary phonons. Furthermore, the atomic disorder might result in some localized phonon modes, similar to ones discussed in a model calculation for $\mathrm{PbTe}^{43}$.

Now how do we interpret the change across $T_{c}$ of the low-energy modes? For a conventional BCS superconductor $\mathrm{Nb}_{3} \mathrm{Sb}$, Axe and Shriane showed that the linewidth for the phonons with energies lower than $2 \Delta$ became

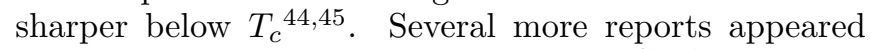
subsequently following these observations 46 52. These results were successfully explained by Allen and coworkers by considering the coupling between conduction electrons and phonons ${ }^{53 / 54}$. In particular, the electrons are gapped below $2 \Delta(T)$. Thus, damping to the phonons caused by electron-phonon interaction vanishes for phonons with energies less than $2 \Delta(T)$, resulting in an increase of the phonon lifetime ${ }^{53154}$. In this case, the total spectral weight of the phonon scattering should be conserved. Our results in Fig. 3 showing that the PDOS increases for $E \leq 2 \Delta$ at low temperatures but no difference is observed for $E>2 \Delta$ are totally unexpected. Nevertheless, this anomalous phonon behavior does indicate the significant electron-phonon coupling known to be present in this class of compounds $14115 / 40$.

In addition, we have In dopants; in the related com-

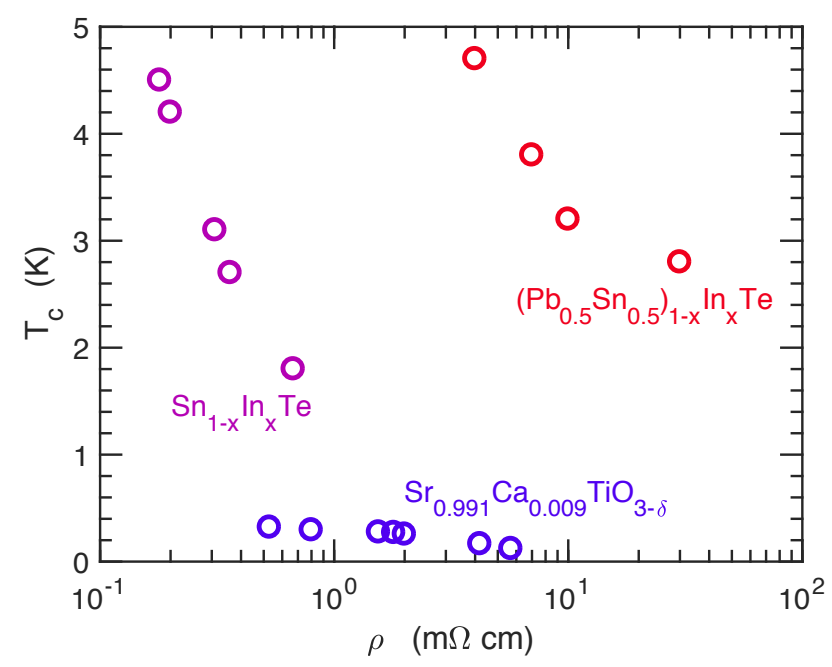

FIG. 5. Comparison of superconducting $T_{c}$ vs. normal-state resistivity, measured just above $T_{c}, \quad$ for $\left(\mathrm{Pb}_{0.5} \mathrm{Sn}_{0.5}\right)_{1-x} \mathrm{In}_{x} \mathrm{Te}^{\sqrt{22}}, \quad \mathrm{Sn}_{1-x} \mathrm{In}_{x} \mathrm{Te}^{26}$, and $\mathrm{Sr}_{0.991} \mathrm{Ca}_{0.009} \mathrm{TiO}_{3-\delta}$. 
pound $\mathrm{Sn}_{1-x} \operatorname{In}_{x} \mathrm{Te}$, each In ion appears to donate one electron, but these electrons only gradually delocalize and become mobile with increasing $x^{55}$. In the present case, the added carriers must be involved in tuning the superconductivity; however, the normal-state resistivity is very large, and has a non-metallic temperature dependence 22127 . To illustrate this, in Fig. 5 we plot $T_{c}$ vs. $\rho$ (measured just above $T_{c}$ ) in PSIT, and compare it with results for $\mathrm{Sn}_{1-x} \mathrm{In}_{x} \mathrm{Te}^{26156}$ and $\mathrm{Sr}_{0.991} \mathrm{Ca}_{0.009} \mathrm{TiO}_{3-\delta}(\mathrm{STO})^{23}$. The carrier density is at least an order of magnitude greater in PSIT than in STO, but the resistivity is much larger in PSIT. We also note that in $\mathrm{Sn}_{1-x} \mathrm{Ag}_{x} \mathrm{Te}$, although the resisitivity just above $T_{c}$ is lower than $0.1 \mathrm{~m} \Omega \mathrm{cm}$, the $T_{c}$ is only about $2 \mathrm{~K}^{[57}$.

When we cool PSIT below $T_{c}$, the presumed quasiparticles that participate in the pairing are expected to decouple from the phonons. If these quasiparticles help to screen the atomic disorder, then decoupling might effectively increase the weight of localized phonons at energies below $2 \Delta$. We are not aware of any previous case where such behavior has been observed. This unusual behavior certainly merits further experimental and theoretical investigations. Of particular interest to understand is whether well-defined quasiparticles exist in the normal state, as they are the starting point for BCS theory $\sqrt{58}$.

In conclusion, we have presented INS results on the phonon spectra of PSIT, with $x=0$, and 0.3. We observe anomalous low-energy features in the PDOS and a substantial enhancement of the phonon intensities below $T_{c}$, only in the superconducting sample, for phonons with energies below almost exactly twice the zero-temperature superconducting gap. While calculations indicate an electron-phonon coupling strength fully compatible with the $T_{c}$, they do not explain the anomalous responses found in $\left(\mathrm{Pb}_{0.5} \mathrm{Sn}_{0.5}\right)_{0.7} \mathrm{In}_{0.3} \mathrm{Te}$. Further study is necessary to understand whether the unusual behavior we have identified is a help or hindrance to the superconducting order.

We thank Xiangang Wan and Phil Allen for stimulating discussions. Work at Nanjing University was supported by the MOST of China (Grant Nos 2016YFA0300404 and 2015CB921202), and NSFC (Grant Nos 11674157, 51372112 and 11574133), NSF Jiangsu province (Grant No. BK20150012), the Fundamental Research Funds for the Central Universities (Grant No. 020414380105), and Special Program for Applied Research on Super Computation of the NSFCGuangdong Joint Fund (the second phase). We also acknowledge the technical support from the HPCC of Nanjing University and "Tianhe-2" at NSCC-Guangzhou, where the calculations were performed. Work at BNL was supported by the Office of Basic Energy Sciences, US Department of Energy under Contract DE-SC0012704. R.D.Z. was supported by the Center for Emergent Superconductivity, an Energy Frontier Research Center, headquartered at BNL, funded by US Department of Energy, under Contract DE-2009-BNL-PM015. Research conducted at ORNL's SNS was sponsored by the Scientific User Facilities Division, Office of Basic Energy Sciences, US Department of Energy.
* Present address: Department of Chemistry, Princeton University, Princeton, New Jersey 08544, USA

$\dagger$ Present address: NIST Center for Neutron Research, National Institute of Standards and Technology, Gaithersburg, Maryland 20899, USA

¡iansun@nju.edu.cn

$\S$ jwen@nju.edu.cn

1 Hasan, M. Z. \& Kane, C. L. Colloquium: Topological Insulators. Rev. Mod. Phys. 82, 3045-3067 (2010).

2 Qi, X.-L. \& Zhang, S.-C. Topological insulators and superconductors. Rev. Mod. Phys. 83, 1057-1110 (2011).

3 Ando, Y. \& Fu, L. Topological Crystalline Insulators and Topological Superconductors: From Concepts to Materials. Annual Review of Condensed Matter Physics 6, 361381 (2015).

4 Fu, L. Topological Crystalline Insulators. Phys. Rev. Lett. 106, 106802 (2011).

${ }^{5}$ Hsieh, T. H., Lin, H., Liu, J., Duan, W., Bansil, A. \& Fu, L. Topological crystalline insulators in the SnTe material class. Nat. Commun. 3, 982 (2012).

6 Xu, S.-Y., Liu, C., Alidoust, N., Neupane, M., Qian, D., Belopolski, I., Denlinger, J.D., Wang, Y.J., Lin, H., Wray, L.A., Landolt, G., Slomski, B., Dil, J.H., Marcinkova, A., Morosan, E., Gibson, Q., Sankar, R., Chou, F.C., Cava, R.J., Bansil, A. \& Hasan, M.Z. Observation of a topological crystalline insulator phase and topological phase transition in $\mathrm{Pb}_{1-x} \mathrm{Sn}_{x}$ Te. Nat. Commun. 3, 1192 (2012).
7 Tanaka, Y., Ren, Z., Sato, T., Nakayama, K., Souma, S., Takahashi, T., Segawa, K. \& Ando, Y. Experimental realization of a topological crystalline insulator in SnTe. Nat. Phys. 8, 800 (2012).

8 Assaf, B. A., Katmis, F., Wei, P., Satpati, B., Zhang, Z., Bennett, S. P., Harris, V. G., Moodera, J. S. \& Heiman, D. Quantum coherent transport in SnTe topological crystalline insulator thin films. Appl. Phys. Lett. 105, 102108 (2014).

9 Akiyama, R., Fujisawa, K., Sakurai, R. \& Kuroda, S. Weak antilocalization in (111) thin films of a topological crystalline insulator SnTe. J. Phys.: Conf. Series 568, 052001 (2014).

10 Sasaki, S., Ren, Z., Taskin, A. A., Segawa, K., Fu, L. \& Ando, Y. Odd-Parity Pairing and Topological Superconductivity in a Strongly Spin-Orbit Coupled Semiconductor. Phys. Rev. Lett. 109, 217004 (2012).

11 He, L. P., Zhang, Z., Pan, J., Hong, X. C., Zhou, S. Y. \& Li, S. Y. Full superconducting gap in the doped topological crystalline insulator $\mathrm{Sn}_{0.6} \operatorname{In}_{0.4}$ Te. Phys. Rev. B 88, 014523 (2013).

12 Saghir, M., Barker, J. A. T., Balakrishnan, G., Hillier, A. D. \& Lees, M. R. Superconducting properties of $\mathrm{Sn}_{1-x} \operatorname{In}_{x} \mathrm{Te}(x=0.38-0.45)$ studied using muon-spin spectroscopy. Phys. Rev. B 90, 064508 (2014).

13 Smylie, M. P., Claus, H., Kwok, W.-K., Louden, E. R., Eskildsen, M. R., Sefat, A. S., Zhong, R. D., Schneeloch, 
J., Gu, G. D., Bokari, E., Niraula, P. M., Kayani, A., Dewhurst, C. D., Snezhko, A. \& Welp, U. Superconductivity, pairing symmetry, and disorder in the doped topological insulator $\operatorname{Sn}_{1-x} \operatorname{In}_{x}$ Te for $x \geq 0.10$. Phys. Rev. B 97, 024511 (2018).

${ }^{14}$ Lucovsky, G. \& White, R. M. Effects of Resonance Bonding on the Properties of Crystalline and Amorphous Semiconductors. Phys. Rev. B 8, 660-667 (1973).

15 Littlewood, P. B. \& Heine, V. The infrared effective charge in IV-VI compounds. I. A simple one-dimensional model. J. Phys. C: Solid State Phys. 12, 4431 (1979).

16 Kobayashi, K. L. I., Kato, Y., Katayama, Y. \& Komatsubara, K. F. Carrier-Concentration-Dependent Phase Transition in SnTe. Phys. Rev. Lett. 37, 772-774 (1976).

17 O'Neill, C. D., Sokolov, D. A., Hermann, A., Bossak, A., Stock, C. \& Huxley, A. D. Inelastic x-ray investigation of the ferroelectric transition in SnTe. Phys. Rev. B 95, 144101 (2017).

18 Daughton, W. J., Tompson, C. W. \& Gurmen, E. Lattice instability and phonon lifetimes in $\mathrm{Pb}_{1-x} \mathrm{Sn}_{x}$ Te alloys. $J$. Phys. C: Solid State Phys. 11, 1573 (1978).

19 Tanaka, Y., Sato, T., Nakayama, K., Souma, S., Takahashi, T., Ren, Z., Novak, M., Segawa, K. \& Ando, Y. Tunability of the $k$-space location of the Dirac cones in the topological crystalline insulator $\mathrm{Pb}_{1-x} \mathrm{Sn}_{x}$ Te. Phys. Rev. B 87, 155105 (2013).

20 Zhong, R., He, X., Schneeloch, J. A., Zhang, C., Liu, T., Pletikosić, I., Yilmaz, T., Sinkovic, B., Li, Q., Ku, W., Valla, T., Tranquada, J. M. \& Gu, G. Surface-statedominated transport in crystals of the topological crystalline insulator In-doped $\mathrm{Pb}_{1-x} \mathrm{Sn}_{x}$ Te. Phys. Rev. B 91, 195321 (2015).

${ }^{21}$ Parfen'ev, R. V., Shamshur, D. V. \& Nemov, S. A. Superconductivity of $\left(\mathrm{Sn}_{1-z} \mathrm{~Pb}_{z}\right)_{1-x} \operatorname{In}_{x}$ Te alloys. Physics of the Solid State 43, 1845-1849 (2001).

${ }^{22}$ Zhong, R. D., Schneeloch, J. A., Shi, X. Y., Xu, Z. J., Zhang, C., Tranquada, J. M., Li, Q. \& Gu, G. D. Superconductivity induced by In substitution into the topological crystalline insulator $\mathrm{Pb}_{0.5} \mathrm{Sn}_{0.5}$ Te. Phys. Rev. B 90, 020505 (2014).

23 Rischau, C. W., Lin, X., Grams, C. P. Finck, D., Harms, S., Engelmayer, J., Lorenz, T., Gallais, Y., Fauqu, B., Hemberger, J. \& Behnia, K. A ferroelectric quantum phase transition inside the superconducting dome of $\mathrm{Sr}_{1-x} \mathrm{Ca}_{x} \mathrm{TiO}_{3-\delta}$. Nat. Phys. 13, 643-648 (2017).

${ }^{24}$ Rowley, S. E., Spalek, L. J., Smith, R. P., Dean, M. P. M., Itoh, M., Scott, J. F., Lonzarich, G. G. \& Saxena, S. S. Ferroelectric quantum criticality. Nat. Phys. 10, 367-372 (2014).

25 Bardeen, J., Cooper, L. N. \& Schrieffer, J. R. Theory of superconductivity. Phys. Rev. 108, 1175 (1957).

26 Zhong, R. D., Schneeloch, J. A., Shi, X. Y., Xu, Z. J., Zhang, C., Tranquada, J. M., Li, Q. \& Gu, G. D. Optimizing the superconducting transition temperature and upper critical field of $\mathrm{Sn}_{1-x} \operatorname{In}_{x}$ Te. Phys. Rev. B 88, 020505 (2013).

27 Zhong, R., Schneeloch, J., Li, Q., Ku, W., Tranquada, J. \& $\mathrm{Gu}, \mathrm{G}$. Indium substitution effect on the topological crystalline insulator family $\left(\mathrm{Pb}_{1-x} \mathrm{Sn}_{x}\right)_{1-y} \mathrm{In}_{y} \mathrm{Te}$ : Topological and Superconducting Properties. Crystals 7, 55 (2017).

28 Tanaka, Y. Tanaka, Y., Sato, T., Nakayama, K., Souma, S., Takahashi, T., Ren, Zhi, Novak, M., Segawa, Kouji \& Ando, Yoichi Tunability of the $k$-space location of the dirac cones in the topological crystalline insulator $\mathrm{Pb}_{1-x} \mathrm{Sn}_{x}$ te.
Phys. Rev. B 87, 155105 (2013).

${ }^{29}$ Winn, B., Filges, U., Garlea, V. O., Graves-Brook, M., Hagen, M., Jiang, C., Kenzelmann, M., Passell, L., Shapiro, S. M., Tong, X. \& Zaliznyak, I. Recent progress on HYSPEC, and its polarization analysis capabilities. EPJ Web Confr. 83, 03017 (2015).

30 Shirane, G., Shapiro, S. M. \& Tranquada, J. M. Neutron Scattering with a Triple-Axis Spectrometer: Basic Techniques (Cambridge University Press, Cambridge, 2002).

31 Du, G., Du, Z. Y., Fang, D. L., Yang, H., Zhong, R. D., Schneeloch, J., Gu, G. D. \& Wen, H. H Fully gapped superconductivity in in-doped topological crystalline insulator $\mathrm{Pb}_{0.5} \mathrm{Sn}_{0.5}$ Te. Phys. Rev. B 92, 020512 (2015).

32 Saghir, M., Barker, J. A. T., Balakrishnan, G., Hillier, A. D. \& Lees, M. R. Superconducting properties of $\mathrm{Sn}_{1-x} \operatorname{In}_{x} \mathrm{Te}(x=0.38-0.45)$ studied using muon-spin spectroscopy. Phys. Rev. B 90, 064508 (2014).

33 Carbotte, J. P. Properties of boson-exchange superconductors. Rev. Mod. Phys. 62, 1027-1157 (1990).

34 Baroni, S., de Gironcoli, S., Dal Corso, A. \& Giannozzi, P. Phonons and related crystal properties from densityfunctional perturbation theory. Rev. Mod. Phys. 73, 515562 (2001).

35 Giannozzi, P. et al. Quantum espresso: a modular and open-source software project for quantum simulations of materials. J. Phys.: Conden. Matter 21, 395502 (2009).

36 Perdew, J. P. \& Zunger, A. Self-interaction correction to density-functional approximations for many-electron systems. Phys. Rev. B 23, 5048-5079 (1981).

37 Bellaiche, L. \& Vanderbilt, D. Virtual crystal approximation revisited: Application to dielectric and piezoelectric properties of perovskites. Phys. Rev. B 61, 7877-7882 (2000).

38 Allen, P. B. \& Dynes, R. C. Transition-temperature of strong-coupled superconductors reanalyzed. Phys. Rev. B 12, 905-922 (1975).

39 Cowley, E. R., Darby, J. K. \& Pawley, G. S. The lattice dynamics of tin telluride. J. Phys. C: Solid State Phys. 2, 1916 (1969).

${ }^{40}$ Li, C. W., Hellman, O., Ma, J., May, A. F., Cao, H. B., Chen, X., Christianson, A. D., Ehlers, G., Singh, D. J., Sales, B. C., \& Delaire, O. Phonon Self-Energy and Origin of Anomalous Neutron Scattering Spectra in SnTe and PbTe Thermoelectrics. Phys. Rev. Lett. 112, 175501 (2014).

41 Pawley, G. S., Cochran, W., Cowley, R. A. \& Dolling, G. Diatomic Ferroelectrics. Phys. Rev. Lett. 17, 753-755 (1966).

42 Cowley, R. A. Temperature Dependence of a Transverse Optic Mode in Strontium Titanate. Phys. Rev. Lett. 9, 159-161 (1962).

43 Zhang, Y., Ke, X., Kent, P. R. C., Yang, J. \& Chen, C. Anomalous Lattice Dynamics near the Ferroelectric Instability in PbTe. Phys. Rev. Lett. 107, 175503 (2011).

44 Axe, J. D. \& Shirane, G. Influence of the superconducting energy gap on phonon linewidths in $\mathrm{Nb}_{3} \mathrm{Sn}$. Phys. Rev. Lett. 30, 214-216 (1973).

45 Axe, J. D. \& Shirane, G. Inelastic-neutron-scattering study of acoustic phonons in $\mathrm{Nb}_{3}$ Sn. Phys. Rev. B 8, 1965-1977 (1973).

46 Shirane, G., Axe, J. \& Shapiro, S. Electron-phonon interaction in superconducting Nb. Solid State Communications 13, 1893 - 1895 (1973).

47 Shapiro, S. M., Shirane, G. \& Axe, J. D. Measurements of 
the electron-phonon interaction in $\mathrm{Nb}$ by inelastic neutron scattering. Phys. Rev. B 12, 4899-4908 (1975).

48 Kawano, H., Yoshizawa, H., Takeya, H. \& Kadowaki, K. Anomalous phonon scattering below $T_{c}$ in $\mathrm{YNi}_{2}{ }^{11} \mathrm{~B}_{2} \mathrm{C}$. Phys. Rev. Lett. 77, 4628-4631 (1996).

${ }^{49}$ Weber, F., Kreyssig, A., Pintschovius, L., Heid, R., Reichardt, W. Reznik, D. Stockert, O. \& Hradil, K. Direct observation of the superconducting gap in phonon spectra. Phys. Rev. Lett. 101, 237002 (2008).

${ }^{50}$ Osborn, R., Goremychkin, E. A., Kolesnikov, A. I. \& Hinks, D. G. Phonon density of states in $\mathrm{MgB}_{2}$. Phys. Rev. Lett. 87, 017005 (2001).

51 Muranaka, T., Margadonna, S., Maurin, I., Brigatti, K., Colognesi, D., Prassides, K., Iwasa, Y., Arai, M., Takata, M. \& Akimitsu, J. Vibrational spectroscopy of superconducting $\mathrm{MgB}_{2}$ by neutron inelastic scattering. J. Phys. Soc. Jpn. 70, 1480-1482 (2001).

${ }^{52}$ Stassis, C., Bullock, M., Zarestky, J., Canfield, P., Goldman, A. I., Shirane, G. \& Shapiro, S. M. Phonon mode coupling in superconducting $\mathrm{LuNi}_{2} \mathrm{~B}_{2}$ C. Phys. Rev. B 55, R8678-R8681 (1997).

53 Allen, P. B. Effect of soft phonons on superconductivity:
A re-evaluation and a positive case for $\mathrm{Nb}_{3} \mathrm{Sn}$. Solid State Communications 14, 937 - 940 (1974).

54 Allen, P. B., Kostur, V. N., Takesue, N. \& Shirane, G. Neutron-scattering profile of $q \neq 0$ phonons in BCS superconductors. Phys. Rev. B 56, 5552-5558 (1997).

55 Zhang, C., He, X. G., Chi, H., Zhong, R., Ku, W., Gu, G., Tranquada, J. M., \& Li, Q. Electron and hole contributions to normal-state transport in the superconducting system $\mathrm{Sn}_{1-x} \mathrm{In}_{x}$ Te. Preprint at https://arxiv.org/abs/1802.09882 (2018).

56 Haldolaarachchige, N., Gibson, Q., Xie, W., Nielsen, M. B., Kushwaha, S. \& Cava, R. J. Anomalous composition dependence of the superconductivity in In-doped SnTe. Phys. Rev. B 93, 024520 (2016).

57 Mizuguchi, Y. \& Miura, O. High-pressure synthesis and superconductivity of Ag-doped topological crystalline insulator SnTe $\left(\mathrm{Sn}_{1-x} \mathrm{Ag}_{x}\right.$ Te with $\left.x=0-0.5\right)$. J. Phys. Soc. Jpn. 85, 053702 (2016).

58 Emery, V. J. \& Kivelson, S. A. Superconductivity in bad metals. Phys. Rev. Lett. 74, 3253 (1995). 